\title{
A computational approach to a doped antiferromagnet: correlations between two spin-polarons in the lightly doped $\mathrm{CuO}_{2}$ plane
}

\author{
Bayo Lau*, ${ }^{*, 1}$ Mona Berciu ${ }^{1,2}$, and George A. Sawatzky ${ }^{1,2}$ \\ * currently at Columbia University, contact at bayo@phys.columbia.edu \\ ${ }^{1}$ Department of Physics and Astronomy, University of British Columbia, Vancouver, British Columbia, V6T $1 Z 1$ and \\ ${ }^{2}$ Quantum Matter Institute, University of British Columbia, Vancouver, British Columbia, V6T 1Z4
}

(Dated: October 26, 2018)

\begin{abstract}
We extend the methods recently introduced in Phys. Rev. Lett. 106036401 (2011) to investigate correlations between two spin-polarons in a quasi-two-dimensional $\mathrm{CuO}_{2}$ layer. The low-energy wavefunctions for two doped holes introduced in a half-filled $\mathrm{CuO}_{2}$ plane with 32 copper and 64 oxygen sites are calculated explicitly using an efficient yet accurate truncation scheme to model the antiferromagnet background. The energetics and wavefucntions show that the charges form three-spin polarons and the spin is carried by a disturbance around the three-spin polaron core. The low-energy band results from the competition between the kinetic energy and a local attractive potential which favors $d_{x^{2}-y^{2}}$ states. Lastly, we point out features that are expected to be robust for larger systems.

PACS numbers: 71.10.Fd,75.10.Jm,71.38.-k,74.72.-h
\end{abstract}

\section{INTRODUCTION}

Spin- $\frac{1}{2}$ antiferromagnetism is of great importance in the quantum description of many exotic materials. Setting as a reference point the exhaustively studied nearestneighbor Heisenberg antiferromagnet (AFM) on the twodimensional square lattice, $\frac{1}{,}$ major developments include studies of frustration due to lattice geometry and to long(er)-range coupling, as well as the disturbance due to additional fermionic charge carriers. Investigations of the latter problem have been strongly driven by the need to understand the doping-controlled evolution of cuprate layers. This is also the focus of this article.

Since their discovery in $1986, \frac{2}{2}$ cuprates have been classified as high- $T_{c}$ superconductors. Their main challenge to condensed matter physics is to understand the basic mechanism leading to high temperature superconductivity, but equally important is the need to understand the many anomalous properties when these materials are tuned away from the superconducting phase. The class of hole-doped cuprates, which allow electron removal from the parent compound, is of particular interest because of the clear separation of the pseudogap regime, in addition to the antiferromagnetism, superconductivity, Fermi liquid and non-Fermi liquid phases which occupy different regions of the phase diagram. The connections between these different phases have not yet been fully elucidated despite many proposals: ${ }^{3-11}$ In fact, one of the few widely accepted ideas in this field is that to find the pairing mechanism will require understanding the various nonsuperconducting phases first.

It is also widely believed that a complete description of the lightly hole-doped spin- $\frac{1}{2} 2 \mathrm{D}$ antiferromagnet (AFM) with full quantum fluctuations could provide clues for understanding the origin of the non-Fermi-liquid behavior and the superconducting ground state observed at higher doping. Consideration of more exotic scenarios $\frac{10,11}{1}$ are exciting developments; however, a detailed modeling of the few holes+AFM problem is a crucial first step needed to appreciate the significance and importance of such additions. This problem is difficult because of the complicated nature of the 2D AFM background, whose quantum fluctuations in the presence of multiple doping holes were never fully captured for a large $\mathrm{CuO}_{2}$ lattice.

Such a theoretical or computational description is challenging because of the strongly-correlated many-body nature of the system. Microscopic hole-AFM interactions have been studied in models with one $\underline{12-19}$ two $\underline{\underline{20}-27}$ three, ${ }^{28-31}$ or more ${ }^{32-38}$ bands. While exact analytical solutions seem to be out of reach, numerical studies are also carried out with various compromises, such as the use of small clusters, variational approaches, and/or modeling of the AFM state as a classical Néel state plus spinwaves $\underline{\underline{39}} \underline{\underline{41}}$ Due to these limitations, there are uncertainties regarding the minimal microscopic model; after all, it took decades to gain a satisfactory understanding of even the simplest one-band t-t'-J model ${ }^{12-14}$ While certain aspects $\underline{42-50}$ of X-ray spectroscopy, Electron Energy Loss Spectroscopy, Scanning Tunneling Microscopy, recent neutron scattering and x-ray absorption measurements cannot be described by one band models, the significance of omitting other bands cannot be quantified without access to solutions of more detailed models.

Cuprates exhibit charge-transfer band-gap behavior with mobile holes located mainly on the anion $2 p$ orbitals and unpaired electrons on cation $3 d$ orbitals,$\underline{51}$ One-band models use superexchange $\mathrm{e}^{\underline{\underline{2}}}$ and Zhang-Rice singlets $(\mathrm{ZRS}) \underline{15}$ to reduce the $(N-n)$-electron problem to one of $n$ holes in an AFM background, in which both spin and charge degrees of freedom are assumed to be hosted in the same single band. To reach agreement with experiments, such models must be tweaked at least by adding longer-range hopping $25-27,53,54$ and possibly by coupling to phonons $11,55,56$.

We have recently shown that even for the one-hole case, distinguishing cation and anion sites leads to significantly 
(a)

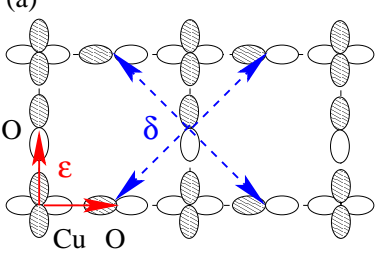

(b)

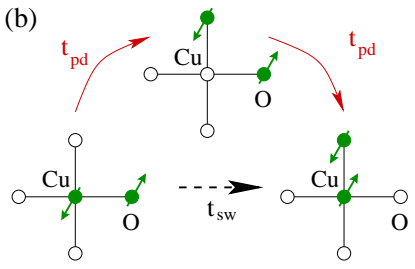

FIG. 1. (color online). (a) Two unit cells of the $\mathrm{CuO}_{2}$ plane. The orbitals kept in the three-band model of Eq. (1) are shown, with white (shaded) for positive (negative) signs. The two $\epsilon$ vectors (solid arrow) and four $\delta$ vectors (dashed arrow) are also shown. (b) Sketch of a virtual process of $T_{\text {swap }}$.

different wavefunctions compared to those of such single band models 57 . In particular, we found that the lowenergy quasiparticle band is a result of the crossing between the bands of spin- $\frac{1}{2}$ and spin- $\frac{3}{2}$ polarons. The $\frac{3}{2}$ polaron has a local spin-1 fluctuation surrounding a spin$\frac{1}{2}$ core. The spectral weight for electron removal is exactly zero at in the region of $k=0$ and $(\pi, \pi)$ because the $\frac{3}{2}$ band crosses below the $\frac{1}{2}$ band. The spectral weight is also exactly zero at $k=(0, \pi)$ even though there is no band crossing. We found that this is due to the orthogonal symmetries of the lowest $k=(0, \pi)$ state and the state created by removing a $k=(0, \pi)$ electron from the AFM GS. These findings prompt a more general study about more doping holes; here we present results for a large cluster with two holes.

The numerical modeling of multi-hole systems at zero temperature is challenging due to the combinatorially large Hilbert space and the fermion sign problem. In this work, we further validate a recently proposed numerical scheme $\underline{58}$ as an efficient and systematic way of modeling the doped AFM relevant to the lightly doped regime. We then use it to calculate the explicit wavefunctions for two holes introduced into a half-filled cluster of 32 copper and 64 oxygen sites with periodic boundary condition. The numerical solution points to a competition between local attractive potential and kinetic energies.

The article is organized as follows: in Section II we introduce our model starting from a general three-band model, and then specify the various assumptions used to simplify it. In Section III we provide computational details and validate our numerical approach. Section IV contains our results. Section V further discusses the results and also points out features that are expected to be robust in larger systems. Section VI contains the conclusions.

\section{THE MODEL}

We start from a three-band $p-d$ model which describes the basic physics of a hole-doped, charge-transfer gap,

insulating spin- $\frac{1}{2}$ antiferromagnet: $\underline{\underline{28}} \underline{\underline{31}}$

$$
\begin{aligned}
H_{3 B}= & T_{p d}+T_{p p}+\Delta_{p d} \sum n_{l+\epsilon, \sigma} \\
& +U_{p p} \sum n_{l+\epsilon, \uparrow} n_{l+\epsilon, \downarrow}+U_{d d} \sum n_{l, \uparrow} n_{l, \downarrow} .
\end{aligned}
$$

Here, $n_{l, \sigma}=d_{l, \sigma}^{\dagger} d_{l, \sigma}$ and $n_{l+\epsilon, \sigma}=p_{l+\epsilon, \sigma}^{\dagger} p_{l+\epsilon, \sigma}$ count holes with spin $\sigma$ in the $\mathrm{Cu} 3 d_{x^{2}-y^{2}}$ orbital at site $l$, respectively the $\mathrm{O} 2 p_{x / y}$ orbital located at $l+\epsilon_{x / y}$, and $U_{d d}>U_{p p}>\Delta_{p d}$ describe Hubbard and charge-transfer interactions.

$$
T_{p d}=t_{p d} \sum\left[\left(-p_{l+\epsilon, \sigma}^{\dagger}+p_{l-\epsilon, \sigma}^{\dagger}\right) d_{l, \sigma}+\text { h.c. }\right]
$$

and

$$
T_{p p}=t_{p p} \sum s_{\delta} p_{l+\epsilon+\delta, \sigma}^{\dagger} p_{l+\epsilon, \sigma}
$$

describe $\mathrm{Cu}-\mathrm{O}$, respectively $\mathrm{O}-\mathrm{O}$ hopping, where the sign $s_{\delta}=\delta_{x} \delta_{y} /\left|\delta_{x} \delta_{y}\right|$ of the hopping matrix elements is determined by the relative phases of the initial and final orbitals. The meaning of the vectors $\epsilon$ and $\delta$ is explained in Fig. 1(a). We have taken the direct $T_{d d}$ hoping to be negligible because of the large spatial separation between $\mathrm{Cu}^{2+}$ ions in the lattice. We have also discarded $T_{p p}^{\prime}$, the direct next nearest neighbor $\mathrm{O}-\mathrm{O}$ hopping, which was found to have negligible effects on the single polaron states found in Ref. 57 and summarized below in Section II.

At half-filling, in the insulating parent compound, states of mainly oxygen $2 p$ character form a completely filled band and there is one hole per $\mathrm{Cu}$. If a no-double occupancy restriction is enforced, the resulting groundstate has AFM order, with a nearest-neighbor $\mathrm{Cu}-\mathrm{Cu}$ superexchange interaction mediated by virtual hopping between oxygen $2 p$ and $\mathrm{Cu} 3 d$ orbitals. An effective model for two doping holes, located at $\mathrm{O}$ sites, interacting with this antiferromagnetic background, can be derived by direct generalization of the $U_{d d} \rightarrow \infty$ Rayleigh-Schrodinger method described in the supplementary material of Ref. 57 for the single hole case. If all hopping integrals are set to zero in the three-band model (Eq. 11), the two-hole GS are of the type:

$$
p_{l+\epsilon, \sigma}^{\dagger} p_{l^{\prime}+\epsilon^{\prime}, \sigma^{\prime}}^{\dagger} \prod_{l^{\prime \prime}}\left|\sigma_{l^{\prime \prime}}\right\rangle_{l^{\prime \prime}},
$$

with each ket $\left|\sigma_{l^{\prime \prime}}\right\rangle_{l^{\prime \prime}}$ specifying the spin of its $\mathrm{Cu}$. These states have a degeneracy of $\left(\begin{array}{c}2 N \\ 2\end{array}\right) 2^{N+2}$. The RayleighSchrodinger expansion operates in this highly degenerate subspace of the $N+2$ hole sector of the Fock space to give an effective Hamiltonian for these states. The outcome is slightly different than in the single-hole scenario. In particular, the projector used in the expansion is

$$
P_{2 h}=\prod\left(1-n_{l+\epsilon, \uparrow} n_{l+\epsilon, \downarrow}\right) \prod\left(n_{l, \uparrow}+n_{l, \downarrow}-2 n_{l, \uparrow} n_{l, \downarrow}\right),
$$

and allows only states with a full lattice of copper spins and no double-occupancies (due to $U_{p p / d d}$ ). In other 
words, the doping holes are forced to live on $\mathrm{O}$ sites. After the expansion, the many resulting terms can be grouped such that the effective Hamiltonian is written as the sum of two parts:

$$
H_{\text {eff }}=P_{2 h} H_{1} P_{2 h}+H_{2} .
$$

$H_{1}$ is the collection of terms when the two holes do not directly affect one another. This single-hole effective Hamiltonian was derived in Ref. 57:

$$
H_{1}=T_{p p}+T_{\text {swap }}+H_{J_{p d}}+H_{J_{d d}},
$$

where the bare oxygen-oxygen hopping of a hole, $T_{p p}$, is supplemented by:

$$
\begin{aligned}
T_{\text {swap }} & =-t_{s w} \sum s_{\eta} p_{l+\epsilon+\eta, \sigma}^{\dagger} p_{l+\epsilon, \sigma^{\prime}}\left|\sigma_{l_{\epsilon, \eta}}^{\prime}\right\rangle\left\langle\sigma_{l_{\epsilon, \eta}}\right| \\
H_{J_{p d}} & =J_{p d} \sum \bar{S}_{l} \cdot \bar{S}_{l \pm \epsilon} \\
H_{J_{d d}} & =J_{d d} \sum \bar{S}_{l \pm 2 \epsilon} \cdot \bar{S}_{l} \Pi_{\sigma}\left(1-n_{l \pm \epsilon, \sigma}\right)
\end{aligned}
$$

The physics described by these terms has been discussed in some detail in the supplementary materials of Ref. 57. Briefly, $H_{J_{p d}}$ is the exchange between the spin of a doping hole located at an $\mathrm{O}$ site, with that of its neighboring $\mathrm{Cu}$ spins. This term favors the formation

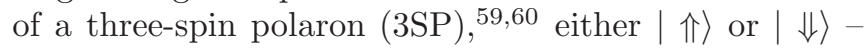
the corresponding eigenfunctions for the central $\mathrm{Cu}-\mathrm{O}$ $\mathrm{Cu}$ spins are listed in Table [1] These describe the ferromagnetic core of the hole-induced disturbance in the otherwise AFM background. $H_{J_{d d}}$ is the usual superexchange between neighboring $\mathrm{Cu}$ spins, which, however, is blocked if a hole is located on the ligand O. Such blocking decreases the penalty for having the 3SP ferromagnetic core in the AFM background. Finally, $T_{\text {swap }}$ describes the processes sketched in Fig. 1(b), where the hole from a $\mathrm{Cu}$ neighbor to the doping $\mathrm{O}$ hole first hops to another of its three hole-free $\mathrm{O}$ neighbors, followed by the original hole falling into the $\mathrm{Cu}$ orbital. The opposite phase between $T_{p p}$ and $T_{s w a p}$ favors the coherent propagation of the three-spin polarons. Even in the single-hole scenario, the physics encompassed by these terms leads to qualitative differences when compared to the t-t'-J model $\stackrel{57}{=}$

Now we identify the important terms contributing to $\mathrm{H}_{2}$, which describes the evolution of the two doping holes when close to each other. We write:

$$
H_{2}=H_{2}^{(2)}+H_{2}^{(3)}+H_{2}^{(4)} \cdots
$$

keeping up to fourth-order terms in the RayleighSchrodinger expansion. In the expansion, the appearance of $\left(1-P_{2 h}\right)$ dictates that all terms must have an even power of $t_{p d}$ because the final states must have one copper spin per unit cell. Because $\mathrm{H}_{2}$ accounts for only

\begin{tabular}{|c|c|c|}
\hline Wavefunction & Total Spin & $\frac{\left\langle H_{J_{p d}}\right\rangle}{J_{p d}}$ \\
\hline$|\Uparrow\rangle=\sqrt{\frac{1}{3}} p_{\uparrow}^{\dagger} \frac{|\uparrow \downarrow\rangle+|\downarrow \uparrow\rangle}{\sqrt{2}}-p_{\downarrow}^{\dagger} \sqrt{\frac{2}{3}}|\uparrow \uparrow\rangle$ & $\frac{1}{2}$ & -1 \\
\hline$|\downarrow\rangle=\sqrt{\frac{1}{3}} p_{\downarrow}^{\dagger} \frac{|\uparrow \downarrow\rangle+|\downarrow \uparrow\rangle}{\sqrt{2}}-p_{\uparrow}^{\dagger} \sqrt{\frac{2}{3}}|\downarrow \downarrow\rangle$ & $\frac{1}{2}$ & -1 \\
\hline$|0+\rangle=\sqrt{\frac{1}{3}} p_{\uparrow}^{\dagger} \frac{|\uparrow \downarrow\rangle-|\downarrow \uparrow\rangle}{\sqrt{2}}$ & $\frac{1}{2}$ & 0 \\
\hline$|0-\rangle=\sqrt{\frac{1}{3}} p_{\downarrow}^{\dagger} \frac{|\uparrow \downarrow\rangle-|\downarrow \uparrow\rangle}{\sqrt{2}}$ & $\frac{1}{2}$ & 0 \\
\hline$\left|\frac{3}{2}, \frac{3}{2}\right\rangle=p_{\uparrow}^{\dagger}|\uparrow \uparrow\rangle$ & $\frac{3}{2}$ & $\frac{1}{2}$ \\
\hline$\left|\frac{3}{2}, \frac{1}{2}\right\rangle=\sqrt{\frac{2}{3}} p_{\uparrow}^{\dagger} \frac{|\uparrow \downarrow\rangle+p_{\downarrow}^{\dagger} \mid \downarrow \uparrow \uparrow}{\sqrt{2}}+\sqrt{\frac{1}{3}} p_{\downarrow}^{\dagger}|\uparrow \uparrow\rangle$ & $\frac{3}{2}$ & $\frac{1}{2}$ \\
\hline$\left|\frac{3}{2},-\frac{1}{2}\right\rangle=\sqrt{\frac{2}{3}} p_{\downarrow}^{\dagger} \frac{|\uparrow \downarrow\rangle+|\downarrow \uparrow\rangle}{\sqrt{2}}+\sqrt{\frac{1}{3}} p_{\uparrow}^{\dagger}|\downarrow \downarrow\rangle$ & $\frac{3}{2}$ & $\frac{1}{2}$ \\
\hline$\left|\frac{3}{2},-\frac{3}{2}\right\rangle=p_{\downarrow}^{\dagger}|\downarrow \downarrow\rangle$ & $\frac{3}{2}$ & $\frac{1}{2}$ \\
\hline
\end{tabular}
2-hole corrections to $P_{2 h} H_{1} P_{2 h}$, one can deduce that all terms in the second-order $H_{2}^{(2)}$ share a factor of $t_{p p}^{2}$. All terms in the third-order $H_{2}^{(3)}$ share a $t_{p d}^{2} t_{p p}$ factor because the second step of any three-step $t_{p p}^{3}$ process would yield virtual excited states projected out by $\left(1-P_{2 h}\right)$.

TABLE I. Single-hole eigenstates of $H_{J_{p d}} \cdot p_{\sigma}^{\dagger}$ creates an oxygen hole and the arrows in the ket indicate the spins of the two copper sites neighboring the oxygen hole.

The fourth-order $H_{2}^{(4)}$ collects terms proportional to $t_{p p}^{4}$, $t_{p p}^{2} t_{p d}^{2}$ or $t_{p d}^{4}$.

The second order corrections are $\frac{t_{p p}^{2}}{U_{p p}}$ processes that link initial and final states in which oxygen holes are $\delta$ apart (Fig. 1a). Because we are considering only the $\sigma$ bonding oxygen orbitals, the virtual excitation is a state with double occupancy of an oxygen orbital; that is, the matrix element is non-zero only for singlet correlations:

$$
H_{2}^{(2)}=\frac{2 t_{p p}^{2}}{U_{p p}} \sum(-1)^{\delta\left(\delta_{1} \cdot \delta_{2}\right)}\left(\bar{S}_{\alpha^{\prime} \alpha} \cdot \bar{S}_{\beta^{\prime} \beta}-\frac{1}{4}\right) p_{l+\epsilon+\delta_{1}, \alpha^{\prime}}^{\dagger} p_{l+\epsilon+\delta_{1}, \alpha} p_{l+\epsilon+\delta_{1}+\delta_{2}, \beta^{\prime}}^{\dagger} p_{l+\epsilon, \beta},
$$

with

$$
\bar{S}_{\alpha^{\prime} \alpha} \cdot \bar{S}_{\beta^{\prime} \beta}=\frac{1}{4} \sum_{i=x, y, z} \sigma_{\alpha^{\prime} \alpha}^{i} \sigma_{\beta^{\prime} \beta}^{i},
$$

where $\sigma_{\alpha^{\prime} \alpha}^{i}$ is an element of the pauli matrix in the $\mathrm{i}^{\text {th }}$ direction. $\delta_{1,2}$ sums over all generic $\delta$ values (Fig. 1h). $\delta\left(\delta_{1} \cdot \delta_{2}\right)=0[1]$ if $\delta_{1}$ and $\delta_{2}$ are parallel [perpendicular]. The matrix element is non-zero only for singlet-like configuration and when the two holes are $|\delta|=\frac{a}{\sqrt{2}}$ apart. There are 8 non-zero matrix elements in this situation. Two of these correspond to $\delta_{1}+\delta_{2}=0$ so the static AFM 
exchange is $2 \times \frac{2 t_{p p}^{2}}{U_{p p}}$. There are also 6 other ways for one hole to "skip" over the other with such a Heisenberg factor.

There is an abundance of terms in third- and fourthorder, which are a sub-set of terms studied in the literature of 2 -band models $\stackrel{20-24}{\underline{2}}$ To provide a simple physical picture, we consider only terms that are greater or equal to $J_{d d}$, which is roughly $\frac{8 \cdot 16 \cdot t_{p p}^{4}}{3 U_{p p}^{3}} \sim \frac{16}{81} t_{p p}$ for $t_{p d} \sim 2 t_{p p}$ and $\Delta_{p d} \sim U_{p p} \sim 6 t_{p p}$. The basis of this approximation is the observation that the dominant short-range physics should be already captured by the numerous low-order terms with an energy scale of $t_{p p}$ and $T_{s w a p}, J_{p d}, J_{p p} \sim$ $0.66 t_{p p}$, which are already greater than the long-range physics at the scale of $J_{d d} \sim 0.2 t_{p p}$. Adding the relevant short-range corrections with magnitude greater than $0.2 t_{p p}$ should then be more than adequate. We also discards terms that can be factored into $T_{p p}, T_{s w a p}, J_{p d}, J_{p p}$ and the identity processes. These terms effectively scale processes by some overall factors which roughly cancel out when all parameters are divided by $J_{d d}$ in order to use dimensionless parameters, since $J_{d d}$ also undergoes similar renormalizations in higher order.

As discussed above, all third-order corrections have a prefactor of $t_{p p} t_{p d}^{2}$. The perturbation goes through two virtual states so the largest possible magnitude is $\frac{t_{p p} t_{p d}^{2}}{\Delta_{p d} U_{p p}} \sim \frac{t_{p p} t_{p d}^{2}}{\Delta_{p d} \Delta_{p d}} \sim \frac{1}{9} t_{p p}$. The splitting due to such a pair of Hermitian matrix elements is $\sim \frac{2}{9} t_{p p}$, of the same order as the $J_{d d}$ splitting. These processes involve virtual excitations with no double occupancy. They are all multiples of $t_{p p}, T_{s w a p}, J_{p d}$ and the identity processes and are discarded due to the rescaling argument discussed above. Because $U_{p p} \sim \Delta_{p d}$, other processes would have denominators that are at least a factor of two greater; that is, constructive quantum interference is required for any terms to be non-negligible compared to the superexchange. Constructive interference among $t_{p p} t_{p d}^{2}$ processes requires the same orbital occupancy in the initial and final state, and this can happen only if the two oxygen holes are $|\delta|$ apart. The transition of interest is then $p_{l \pm e_{x}, \alpha}^{\dagger} p_{l \pm e_{y}, \beta}^{\dagger} d_{l, \gamma}^{\dagger} \rightarrow p_{l \pm e_{x}, \alpha^{\prime}}^{\dagger} p_{l \pm e_{y}, \beta^{\prime}}^{\dagger} d_{l, \gamma^{\prime}}^{\dagger}$. The effective matrix elements have the form $\left\langle\alpha^{\prime}, \beta^{\prime}, \gamma^{\prime}\left|H_{2}^{(3)}\right| \alpha, \beta, \gamma\right\rangle$, a local three-spin ring involving the copper spin sandwiched by the oxygen holes. The correction term can be expressed as a summation over each copper spin with two additional vector $\Delta_{x / y}$ summed over $\pm \epsilon_{x / y}$. Noting that the 3 -step hopping would give an overall phase of -1 (Fig. 1. a ) and that the virtual states have doubleoccupancy, it is not surprising that all operators contain a shifted Heisenberg factor:

$$
\begin{aligned}
H_{2}^{(3)}=\frac{2 t_{d p}^{2} t_{p p}}{U_{p p}\left(\Delta_{p d}+U_{p p}\right)} \sum & \left(\bar{S}_{\beta^{\prime} \beta} \cdot \bar{S}_{\alpha^{\prime} \alpha}-\frac{1}{4}\right) p_{l+\Delta_{x}, \beta^{\prime}}^{\dagger} p_{l+\Delta_{x}, \alpha} p_{l+\Delta_{y}, \gamma}^{\dagger} p_{l+\Delta_{y}, \beta}\left|l, \alpha^{\prime}\right\rangle\langle l, \gamma| \\
+ & \left(\bar{S}_{\alpha^{\prime} \alpha} \cdot \bar{S}_{\beta^{\prime} \beta}-\frac{1}{4}\right) p_{l+\Delta_{x}, \gamma}^{\dagger} p_{l+\Delta_{x}, \alpha} p_{l+\Delta_{y}, \alpha^{\prime}}^{\dagger} p_{l+\Delta_{y}, \beta}\left|l, \beta^{\prime}\right\rangle\langle l, \gamma| \\
+ & \left(\bar{S}_{\gamma^{\prime} \gamma} \cdot \bar{S}_{\alpha^{\prime} \alpha}-\frac{1}{4}\right) p_{l+\Delta_{x}, \gamma^{\prime}}^{\dagger} p_{l+\Delta_{x}, \alpha} p_{l+\Delta_{y}, \alpha^{\prime}}^{\dagger} p_{l+\Delta_{y}, \beta}|l, \beta\rangle\langle l, \gamma| \\
+ & \left(\bar{S}_{\beta^{\prime} \beta} \cdot \bar{S}_{\gamma^{\prime} \gamma}-\frac{1}{4}\right) p_{l+\Delta_{x}, \beta^{\prime}}^{\dagger} p_{l+\Delta_{x}, \alpha} p_{l+\Delta_{y}, \gamma^{\prime}}^{\dagger} p_{l+\Delta_{y}, \beta}|l, \alpha\rangle\langle l, \gamma| \\
+\frac{2 t_{d p}^{2} t_{p p}}{\left(\Delta_{p d}+U_{p p}\right)^{2} \sum} & \delta_{\alpha \beta}\left(\bar{S}_{\beta^{\prime} \beta} \cdot \bar{S}_{\gamma^{\prime} \gamma}-\frac{1}{4}\right) p_{l+\Delta_{x}, \alpha}^{\dagger} p_{l+\Delta_{x}, \alpha} p_{l+\Delta_{y}, \beta^{\prime}}^{\dagger} p_{l+\Delta_{y}, \beta}\left|l, \gamma^{\prime}\right\rangle\langle l, \gamma| \\
+ & \delta_{\gamma \beta}\left(\bar{S}_{\alpha^{\prime} \alpha} \cdot \bar{S}_{\beta^{\prime} \beta}-\frac{1}{4}\right) p_{l+\Delta_{x}, \gamma}^{\dagger} p_{l+\Delta_{x}, \alpha} p_{l+\Delta_{y}, \alpha^{\prime}}^{\dagger} p_{l+\Delta_{y}, \beta}\left|l, \beta^{\prime}\right\rangle\langle l, \gamma| \\
+ & \delta_{\gamma \alpha}\left(\bar{S}_{\beta^{\prime} \beta} \cdot \bar{S}_{\alpha^{\prime} \alpha}-\frac{1}{4}\right) p_{l+\Delta_{x}, \beta^{\prime}}^{\dagger} p_{l+\Delta_{x}, \alpha} p_{l+\Delta_{y}, \gamma}^{\dagger} p_{l+\Delta_{y}, \beta}\left|l, \alpha^{\prime}\right\rangle\langle l, \gamma| \\
+ & \delta_{\beta \alpha}\left(\bar{S}_{\alpha^{\prime} \alpha} \cdot \bar{S}_{\gamma^{\prime} \gamma}-\frac{1}{4}\right) p_{l+\Delta_{x}, \alpha^{\prime}}^{\dagger} p_{l+\Delta_{x}, \alpha} p_{l+\Delta_{y}, \beta}^{\dagger} p_{l+\Delta_{y}, \beta}\left|l, \gamma^{\prime}\right\rangle\langle l, \gamma|
\end{aligned}
$$

These terms are finite if there is a 2-spin singlet amongst the 3 spins. The first four terms give an eigenvalue of $+4 \frac{t_{d p}^{2} t_{p p}}{U_{p p}\left(\Delta_{p d}+U_{p p}\right)} \sim 0.2 t_{p p}$ for oxygen-oxygen singlet pair and 0 for triplet pair. The last four terms give an eigenvalue of $\frac{t_{d p}^{2} t_{p p}}{\left(\Delta_{p d}+U_{p p}\right)^{2}} \sim 0.02 t_{p p}$ for singlet pairs and $0,-3 \frac{t_{d p}^{2} t_{p p}}{\left(\Delta_{p d}+U_{p p}\right)^{2}} \sim-0.06 t_{p p}$ for triplets. Therefore we can approximate this term by simply raising the energy of oxygen-oxygen singlet pairs accordingly.

In the fourth-order, all $t_{p d}^{4}$ processes can be factored into two $T_{\text {swap }}$ or $J_{p d}$ processes. The $t_{p d}^{2} t_{p p}^{2}$ processes are smaller than $J_{d d}$ by a factor of $\sim 4 \times 4$ and no constructive interference is possible. $2 t_{p p}^{4}$ processes are even smaller. Therefore, we set $H_{2}^{(4)} \approx 0$. In summary, the 2-hole 
correction is in essence:

$$
\begin{aligned}
H_{2} \approx & +J_{p p}^{(2)} \sum(-1)^{\delta\left(\delta_{1} \cdot \delta_{2}\right)}\left(\bar{S}_{\alpha^{\prime} \alpha} \cdot \bar{S}_{\beta^{\prime} \beta}-\frac{1}{4}\right) p_{l+\epsilon+\delta_{1}, \alpha^{\prime}}^{\dagger} p_{l+\epsilon+\delta_{1}, \alpha} p_{l+\epsilon+\delta_{1}+\delta_{2}, \beta^{\prime}}^{\dagger} p_{l+\epsilon, \beta} \\
& -J_{p p}^{(3)} \sum\left(\bar{S}_{\alpha^{\prime} \alpha} \cdot \bar{S}_{\beta^{\prime} \beta}-\frac{1}{4}\right) p_{l+\epsilon+\delta, \alpha^{\prime}}^{\dagger} p_{l+\epsilon+\delta, \alpha} p_{l+\epsilon, \beta^{\prime}}^{\dagger} p_{l+\epsilon, \beta}
\end{aligned}
$$

with $J_{p p}^{(2)}=\frac{2 t_{p p}^{2}}{U_{p p}}$ and $J_{p p}^{(3)}=\frac{4 t_{d p}^{2} t_{p p}}{U_{p p}\left(\Delta_{p d}+U_{p p}\right)}$ for hole-hole interaction due to second and third order corrections. Using $t_{p d}=1.3 \mathrm{eV}, t_{p p}=0.65 \mathrm{eV}, \Delta_{p d}=3.6 \mathrm{eV}$, and $U_{p p}=4 e V, 12$ we scale the parameters in units of $J_{d d}$ to find their dimensionless values to be $t_{p p}=4.13, t_{s w}=$ $2.98, J_{p d}=2.83$, and $J_{p p}^{(2)}=1.3420$, and $J_{p p}^{(3)}=0.9182$.

\section{THE COMPUTATION}

Although the thermodynamic limit is important in condensed matter theory, models detailing the interactions between fermionic carriers and a quantum antiferromagnet present formidable challenges to analytical solutions. Finite cluster numerical calculations are therefore a valuable tool in extracting information about the model of interest. Numerical studies of phase transitions would require $N \rightarrow \infty$ extrapolation; instead, this work focuses on the correlations between two holes injected in a half-filled $N=32 \mathrm{CuO}_{2}$ cluster with superior topological properties compared to smaller clusters 61 The computational breakthrough described in this section allows us to explicitly obtain the low-energy wavefunctions. We can then calculate any correlators of interest, in order to understand the nature of these eigenstates. We can also perform a direct comparison with the results of the t-t'-J model with two holes on the same $N=32$ cluster. 19

\section{A. An insight about antiferromagnets}

Even after exploiting translational and spin-projection symmetries, two holes injected into a half-filled $N=32$ $\mathrm{CuO}_{2}$ cluster have a Hilbert space with $0.154 \times 10^{12}$ states. A system of this size challenges the capability of all unbiased methods at zero temperature. One way forward is to identify how to drastically reduce this dimension while keeping the most important states in the basis. The two doping holes contribute at most a factor of $4\left(\begin{array}{c}2 N \\ 2\end{array}\right)$, so it is the AFM background that is primarily responsible for this large number.

We have previously proposed an efficient numerical approach for the modeling of antiferromagnets,, 58 upon which we will build here. For completeness, we first briefly review the undoped scenario 58 - here the system is described by AFM Heisenberg superexchange between neighbor $\mathrm{Cu}$ spins, see $\mathrm{Eq}$. (8). If the $\mathrm{Cu}$ spins on the square lattice are divided into two sublattices, A and B, such that spins from each sublattice only couple to those of the other sublattice, one of the many measures of the staggered magnetization can be expressed in terms of the total spin $S=S_{A}+S_{B}$ :

$$
\hat{m}^{2}=\frac{1}{N^{2}}\left(\sum_{r}(-1)^{|r|} \hat{S}_{r}\right)^{2}=\frac{1}{N^{2}}\left(2 \hat{S}_{A}^{2}+2 \hat{S}_{B}^{2}-\hat{S}^{2}\right) .
$$

The undoped GS is known to be a singlet, $S=0$. For $\hat{S}_{A}$ and $\hat{S}_{B}$ to add to zero, their quantum numbers must be equal, $S_{A}=S_{B}$. Moreover, there are accurate estimates of $m$, which range from $\sim 0.3$ as $N \rightarrow \infty$ to $\sim 0.45$ for $N=32 . \underline{1}$ In order to get such large values for $m$, the sublattice spins $S_{A / B}$ have to be within $\frac{N}{16}$ of their maximum values. In other words, in each sublattice $\frac{N}{8}$ spins add to a total spin of zero while the rest add to the maximum $3 \frac{N}{16}$. Based on this insight, we showed that the GS can be captured systematically by considering a subspace specified by a completeness parameter

$$
C_{S} \in[0,1]
$$

The subspace equals the full Hilbert space when $C_{S}=1$. For smaller values of $C_{S}$, only the states with

$$
S_{A / B} \geq \frac{N}{4}\left(1-C_{S}\right)
$$

are included. $C_{S}=0$ is, therefore, a singlet containing the classical Néel state. A linear decrease of $C_{S}$ yields a combinatorial decrease in the number of states. In Ref. 58 we postulated apriorily that the subspace with $C_{S}=\frac{1}{4}$ captures the essence of the wavefunction, and then showed that the convergence is exponential as $C_{S}$ is tuned from zero to unity and that $C_{S}=\frac{1}{4}$ is indeed the "sweet spot".

For a large sample, two additional holes cannot drastically change the entire AFM background; therefore, the stable, systematic convergence of the undoped AFM is expected to hold for the two-hole scenario.

We first test the method against the exactly solvable scenario of one hole on a cluster with $32 \mathrm{Cu}$ and 64 $\mathrm{O} . \frac{57}{7}$ Using the same truncation criterion, we calculate the one-hole ground state for increasing $C_{S}$. The convergence is illustrated in Fig. 2] The energy computed for $C_{S}=\frac{1}{4}$ and $\frac{1}{2}$ is within $3.6 \%$ and $0.5 \%$, respectively, 

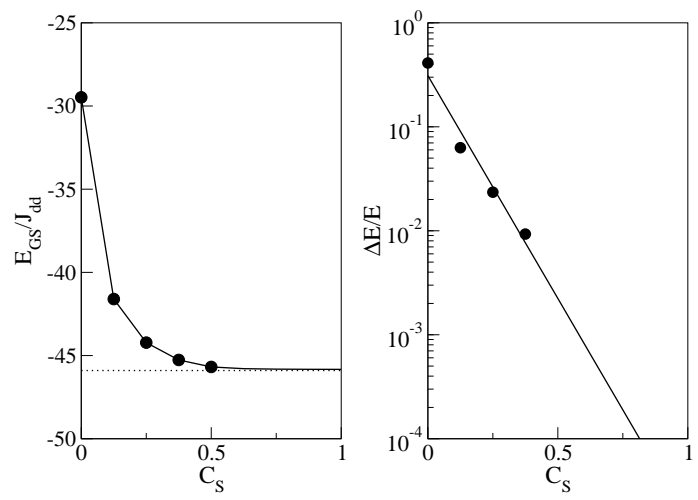

FIG. 2. Convergence of the one-hole ground state energy. Left: GS energy calculated for increasing $C_{S}$. The value approaches rapidly the exact value marked by the dotted line. Right: fractional change in the GS energy for the next increment of $C_{s}$. Solid lines are extrapolation and linear fits.

\begin{tabular}{c|rrrrrr|rrr}
$S_{A} \backslash{ }^{S_{B}}$ & 0 & 1 & 2 & 3 & 4 & 5 & 6 & 7 & 8 \\
\hline 0 & 0.00 & 0.00 & 0.00 & 0.00 & 0.00 & 0.00 & 0.00 & 0.00 & 0.00 \\
1 & 0.00 & 0.02 & 0.03 & 0.00 & 0.00 & 0.00 & 0.00 & 0.00 & 0.00 \\
2 & 0.00 & 0.03 & 0.14 & 0.16 & 0.00 & 0.00 & 0.00 & 0.00 & 0.00 \\
3 & 0.00 & 0.00 & 0.16 & 0.69 & 0.68 & 0.00 & 0.00 & 0.00 & 0.00 \\
4 & 0.00 & 0.00 & 0.00 & 0.68 & 2.52 & 2.21 & 0.00 & 0.00 & 0.00 \\
5 & 0.00 & 0.00 & 0.00 & 0.00 & 2.21 & 7.06 & 5.28 & 0.00 & 0.00 \\
\hline 6 & 0.00 & 0.00 & 0.00 & 0.00 & 0.00 & 5.28 & 14.24 & 8.44 & 0.00 \\
7 & 0.00 & 0.00 & 0.00 & 0.00 & 0.00 & 0.00 & 8.44 & 18.06 & 6.85 \\
8 & 0.00 & 0.00 & 0.00 & 0.00 & 0.00 & 0.00 & 0.00 & 6.85 & 9.97
\end{tabular}

TABLE II. ( $\mathrm{N}=32)$ The $\mathrm{S}=\frac{1}{2} k=\left(\frac{\pi}{2}, \frac{\pi}{2}\right)$ single-hole ground state's probability in subspaces of particular $S_{A} \otimes S_{B}$ values. Numbers are percentages adding up to $100 \%$. Two lines are drawn to highlight the $C_{S}=\frac{1}{4}$ truncation which discards states with $S_{A}, S_{B}<6$ and yields an energy within $3.6 \%$ of the exact value.

of the exact value. Table $\Pi$ shows the exact wavefunction's probability in various subspaces $S_{A} \otimes S_{B}$ of the sublattice total spins $S_{A / B}$. While the $C_{S}=\frac{1}{4}$ truncation captures $\sim 95 \%$ of the probability of the undoped wavefunction,,$\frac{58}{=}$ Table $\llbracket$ shows that $C_{S}=\frac{1}{4}$ captures $\sim$ $73 \%$ of the one-hole groundstate. The next two increments of $C_{S}$ contains $17 \%$ and $7 \%$, respectively, of the remaining weight. The addition of a hole couples the spin background to adjacent values of $S_{A} \otimes S_{B}$, and states added by increasing $C_{S}$ have decreasing importance in the wavefunction; therefore, it is reasonable to expect an increment of $\delta C_{S} \sim \frac{1}{N}$ from $C_{S}=\frac{1}{4}$ to suffice. At least in the very dilute limit, the truncation scheme provides a good starting point to systematically capture the low energy state.

These observations provide reasonable merits for the application of this scheme to the two-hole scenario, whose ground state should be captured in the subspace of $C_{S} \sim \frac{1}{4}+O\left(\delta C_{S}\right)$ for large $N$. To provide a conservative error analysis, however, we would aim for a capability of up to $C_{S} \sim \frac{1}{2}$, which in turn limits the cluster size to $N=32$. We showed in our previous work ${ }^{57}$ that a single oxygen hole induces a local disturbance in the AFM background and that, for the parameter range of interest, the disturbance affects $\sim 6-12$ spins around the hole. Therefore, a cluster with 32 unit cells should be large enough to accommodate two holes without artificially forcing them too close together. As discussed below, our results are different from those yielded by similar attempts restricted to $N=16$ unit cells $\underline{\underline{20}-24}$

\section{B. Implementation}

The Hilbert space of the two-hole problem (Eq. 4 to-

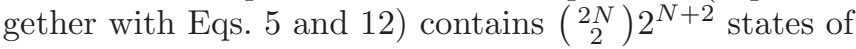
the form $p_{l+\epsilon, \sigma}^{\dagger} p_{l^{\prime}+\epsilon^{\prime}, \sigma^{\prime}}^{\dagger} \prod\left|\sigma_{l^{\prime \prime}}\right\rangle$, with $l+\epsilon \neq l^{\prime}+\epsilon^{\prime}$. To perform computations for large $N$, this basis must be reorganized to take advantage of translational symmetry, total-spin symmetry, total-spin-projection symmetry, and, most importantly, the truncation scheme discussed in the previous section. The implementation is detailed in Appendix A.

We emphasize that the truncation scheme is applied only to the AFM background, i.e. to the parts of the wavefunction describing the spins at $\mathrm{Cu}$ sites. There is no restriction for the two doping holes residing on the $\mathrm{O}$ sites, apart from no-double occupancy.

\section{RESULTS}

We apply the approach described above to solve the two doping holes problem for the $N=32$ cluster. We find that the low-energy states have a total spin of $S_{T}=0$ or $S_{T}=1$ (the total spin includes both the contribution of the AFM background and of the doping holes). Sectors with higher $S_{T}$ have higher energies, in accordance with the trend of finite size AFM computations. 1 As discussed in Ref. 57, the one-hole GS are degenerate at $k=\left( \pm \frac{\pi}{2}, \pm \frac{\pi}{2}\right)$, so the important total momenta for two holes are $K=(0,0),(\pi, 0),(\pi, \pi)$. The convergence of the lowest states at these high-symmetry points is shown in Fig. 3. The trend of exponential convergence is similar to that found for the undoped $\stackrel{58}{ }$ and single hole cases (Fig. 22), signaling that the dominant part of the Hilbert space has been captured. We first present the energetics then illustrate the numerical eigenvectors in details.

\section{A. Energetics}

Figure 4 shows the dispersion of the lowest two-hole states versus the total momentum $K$, for $S_{T}=0,1$. In the $S_{T}=0$ sector, the $K=(\pi, \pi)$ global groundstate is doubly degenerate. The $K=(\pi, 0)$ and $K=(0, \pi)$ states are $\sim 0.17 J_{d d}$ higher in energy, while the $K=(0,0)$ state 

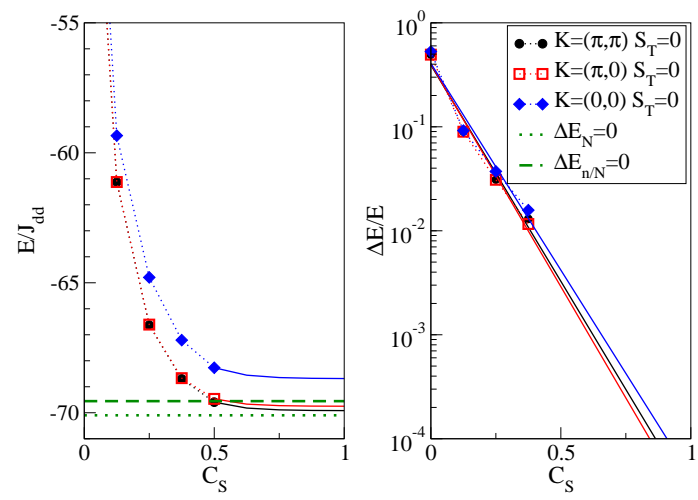

FIG. 3. Convergence of two-hole low energy states at several high-symmetry points. The horizontal dashed and dotted lines are the $\Delta E_{n / N}=0$ and $\Delta E_{N}=0$ levels, respectively, i.e. zero binding energy levels calculated at fixed doping concentration and fixed lattice size, respectively. The two values bound the binding energy value and converge as $N \rightarrow \infty$.

is almost $2 J$ higher in energy. The shape of the energy dispersion mirrors that of some previous studies treating $N \geq 32$ systems with two holes $\frac{19,62}{}$ Although finitemomentum superconducting pair has been proposed by Fulde, Ferrell, Larkin, and Ovchinnikov ${ }^{63,64}$, this scenario is not superconducting. For $N=16$, the GS is instead found to be located at $K=(\pi, 0) \stackrel{24}{\underline{24}}$ This signals that $N=16$ is definitely too small to capture the twohole physics, which is not surprising since a single polaron was found to disturb $\sim 6-12$ spins in its vicinity 57

As for the $S_{T}=1$ sector, these states cross below the $S_{T}=0$ states in the region around $K=0$. The lowest $S_{T}=1, K=0$ level is doubly degenerate as well. Its energy is $\sim 0.3 J_{d d}$, equal to the $\sim \frac{1}{N}$ free magnon gap of the undoped $N=32$ cluster $\stackrel{1}{=}$ The following section confirms that these $S_{T}=1, K=0$ low energy states have the two holes in their doubly degenerate global GS with $S_{T}=0, K=(\pi, \pi)$, plus a $Q=(\pi, \pi), S=1$ "magnon"like excitation in the AFM background. It is thus reasonable to expect the $S_{T}=1, K=0$ states to become degenerate with the $K=(\pi, \pi), S_{T}=0$ states as $N \rightarrow \infty$ and the free magnon gap closes. Similarly, the low energy $S_{T}=1$ excitations at $K=(\pi, 0)$ and $K=(0, \pi)$ are the $S_{T}=0$ two-hole state at $K=(0, \pi)$, respectively $K=(\pi, 0)$, plus a $Q=(\pi, \pi), S=1$ "magnon". They are also expected to become degenerate as $N \rightarrow \infty$.

For the one-hole case, we were able to establish the robustness of spin-1 excitations because we found that $E_{3 / 2}-E_{1 / 2}$ is much lower than any finite-size spin excitation, $E_{\text {magnon }}-E_{1 / 2}{ }^{57}$ In the two-hole case, however, all spin excitations are higher than $E_{\text {magnon }}-E_{0}$, and no conclusions can be drawn.

We note that the two-hole bandwidth is $\sim 2 J_{d d}$, which is roughly equal to the single-polaron bandwidth 57 This suggests that the GS has very weak, if any, binding. (The $\sim 2 J_{d d}$ scale has further implication when the wavefunction is considered in Sec. IV.) The binding energy is the

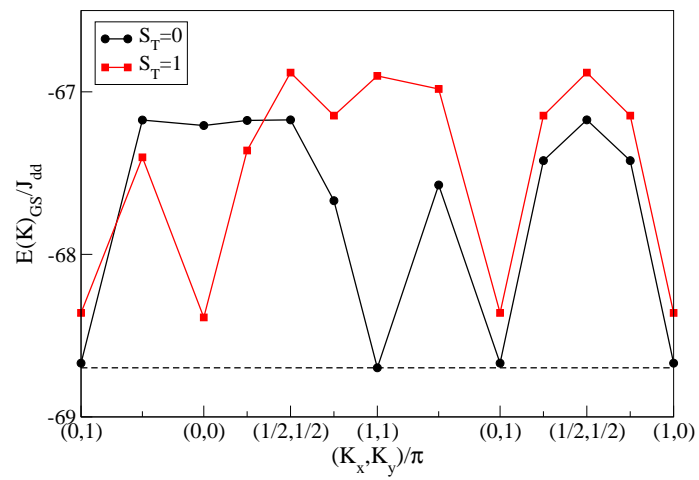

FIG. 4. Energy of the lowest two-hole states vs total momentum along high-symmetry cuts, for $S_{T}=0,1$ and $C_{S}=\frac{3}{8}$.

difference between the two-hole energy and twice the onehole energy, shifted by the energy of the undoped system:

$$
\Delta E=\left(E_{2 h}-E_{0 h}\right)-2\left(E_{1 h}-E_{0 h}\right) .
$$

This value must be extracted with care for a finite cluster. Although the $N=32$ cluster can accommodate two spin polarons, and each disturbs $\sim 6-12 \mathrm{Cu}$ spins in its vicinity,$\stackrel{57}{=}$ as we show in the next section we find that the low energy states have a low probability to be close enough to share a common $\mathrm{Cu}$. Unlike the $N \rightarrow \infty$ limit, the local range of this blockade is not negligible in a $N=$ 32 cluster so the kinetic energy is over-estimated. The one-hole scenario does not have this artifact, leading to an overestimation if the value is evaluated from energies calculated at a constant $N$,

$$
\Delta E_{N}=\left(E_{2 h}^{(32)}-E_{0 h}^{(32)}\right)-2\left(E_{1 h}^{(32)}-E_{0 h}^{(32)}\right) .
$$

Given that $N$-dependent scaling is impossible because $N=16$ clusters have a different ground state (e.g. at momentum $(0, \pi)$ instead of $(\pi, \pi)) \stackrel{24}{\underline{w}}$ and that a larger cluster cannot currently be solved, we estimate a reasonable lower bound for the binding energy by considering the one- and two-hole energies at fixed concentration $\frac{n}{N}=\frac{2}{32}=\frac{1}{16}$,

$$
\Delta E_{\frac{n}{N}}=\left(E_{2 h}^{(32)}-E_{0 h}^{(32)}\right)-2\left(E_{1 h}^{(16)}-E_{0 h}^{(16)}\right) .
$$

These definitions converge in the large $N$ limit,

$$
\Delta E=\lim _{N \rightarrow \infty} \Delta E_{N}=\lim _{N \rightarrow \infty} \Delta E_{\frac{n}{N}},
$$

and thus give the upper- and lower-bound for the $N=32$ cluster.

As shown in Fig. 3. the GS energy was extrapolated to be $E_{2 h}^{(32)}=-69.917 J_{d d}$. The $\Delta E_{N}=0$ and $\Delta E_{\frac{n}{N}}=0$ levels in the same figure indicate a binding energy of $(-0.091 \pm 0.272) J_{d d}$. This suggests weak, if any, binding, although solutions for larger clusters are required to reduce the error bars sufficiently to be able to draw a rigorous conclusion. 


\section{B. Wavefunction analysis}

\section{Symmetry}

We define $P_{x y / \underline{x} y}$ for reflections about the diagonals. The two degenerate $S=0, K=(\pi, \pi)$ GSs were found to have $P_{x y}=-P_{\underline{x} y}= \pm 1$. The two degenerate $S=1$, $K=(0,0)$ states related to them by a gapless $Q=(\pi, \pi)$ magnon were also found to have $P_{x y}=-P_{\underline{x} y}= \pm 1$. This is $p$-wave parity, but it is not a concern because we are outside of the superconducting region. For example, the product of two $P_{x y}=-P_{\underline{x} y}= \pm 1$ wavefunctions would yield a $d_{x^{2}-y^{2}}$ symmetry. Defining $P_{x / y}$ for reflections about the lattice parameter directions, the $K=(0, \pi)$ state was found to have $p_{y}$ symmetry and $K=(\pi, 0)$ state has $p_{x}$ symmetry as in the t-t'-J model.

Unlike in the $N=32$ t-t'-J model whose lowest twohole $S=0, K=0$ state has s-symmetry $\underline{19}$, the lowest $S=0, K=0$ state has $P_{x y}=P_{\underline{x} y}=-1$, which is $d_{x^{2}-y^{2}}$ symmetry. Note that the lowering of s-symmetric state in the t-t'-J model is due to the t' hopping of the Zhang-Rice singlet, and the t' hopping is included in this work due to the explicit consideration of oxygen-oxygen hopping.

\section{Charge correlations}

The charge correlation as a function of the separation $R$ between the two doping holes can be characterized by the expectation value of:

$$
\begin{aligned}
\hat{c}(\eta) & =\sum_{l, \epsilon, \sigma, \sigma^{\prime}} n_{l+\epsilon, \sigma} n_{l+\epsilon+\eta, \sigma^{\prime}} \\
\hat{C}(R) & =\sum_{|\eta|=R} \hat{c}(\eta)
\end{aligned}
$$

with $\sum_{R} \hat{C}(R)=1$. On a finite cluster, the number of hole-hole configuration separated by a distance $R>$ $\frac{L}{2}$ is limited by the periodic boundary condition to be smaller than in the $L^{2}=N \rightarrow \infty$ limit. Therefore, such correlations should be compared to the probability $P(R)$ of two randomly distributed oxygen holes to be separated by $R$ in the same finite cluster. Then, the correlations can be gauged by

$$
\Delta \hat{C}(R)=\hat{C}(R)-P(R),
$$

meaning that the correlation is the same as that of a random distribution if $\Delta C(R)=\langle\Delta \hat{C}(R)\rangle=0$.

The expectation values $C(R)=\langle\hat{C}(R)\rangle$ are shown in Fig. [at various high-symmetry points. The lowest states with $K=(\pi, \pi) S_{T}=0$ and $K=(0,0) S_{T}=1$ have the same $C(R)$, confirming that they are indeed linked by a $Q=(\pi, \pi)$ magnon excitation.

$\Delta C(R)$ features a monotonic increase with $\mathrm{R}$ for the GS, a small peak of correlation at $R=2 a$ for $(\pi, 0)$, and
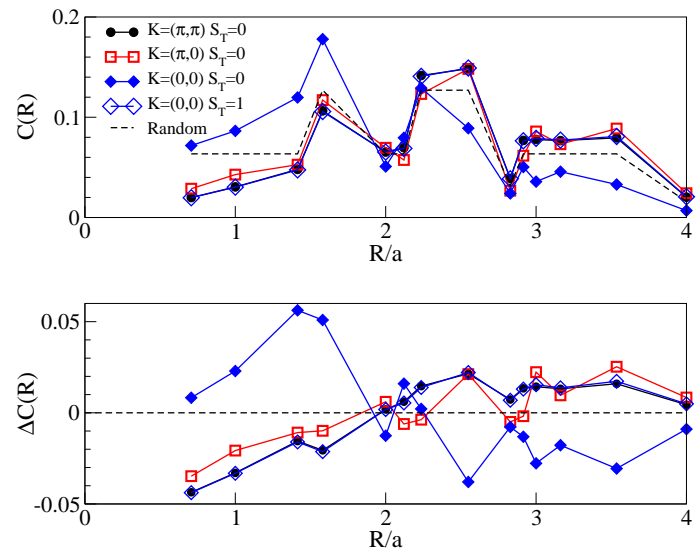

FIG. 5. Top: Probability of charge separation, $\langle C(R)\rangle$ for the lowest state in the $C_{S}=\frac{1}{2}$ subspace. Bottom: The difference from a random distribution. See text for details.

local attraction for the $S=0 K=(0,0)$ state. Section IV.A shows that the $\sim 2 J_{d d}$ single-polaron bandwidth is the energy difference between the GS with extended $\Delta C(R)$ and the $K=(0,0) d_{x^{2}-y^{2}}$ state with local $\Delta C(R)$. The low energy two-particle band is thus a result of the kinetic energy competing with an attractive energy which induces a local $d_{x^{2}-y^{2}}$ pair.

\section{Polaronic Nature}

In the single-hole case, $\stackrel{57}{=}$ at low-energies the mobile carrier is well described as the spin- $\frac{1}{2} 3$ SP's, $|\Uparrow\rangle$ and $|\Downarrow\rangle$ in Table \. which are correlated states of the oxygen spin with its two neighboring copper spins. For the exact ground state $\left\langle H_{J_{p d}}\right\rangle \sim-0.9 J_{p d}$, close to the $-J_{p d}$ energy of the exact 3SP. The two-hole solutions yields $\left\langle H_{J_{p d}}\right\rangle \sim-1.8 J_{p d}$, showing that thinking in terms of $3 \mathrm{SP}$ is still valid and fruitful. In this scenario, the oxygen holes could neighbor the same copper spin to form a 5 -spin object, but $C(R)$ of Fig. 5 shows that the probability for this is low. Ignoring these shared-copper configurations, the wavefunction contains two polarons involving a total of six spins. Noting that $\left\langle H_{J_{p d}}\right\rangle \sim-1.8 J_{p d}$, the single-polaron levels in Tab. [ suggest that the dominant part of the wavefunction contains four possible 3SP pairs with $\frac{\left\langle H_{J_{p d}}\right\rangle}{J_{p d}}=-2: \quad|\Uparrow\rangle|\Downarrow\rangle,|\Uparrow\rangle|\Downarrow\rangle,|\Uparrow\rangle|\Uparrow\rangle$, and $|\Downarrow\rangle|\Downarrow\rangle$. Other pair configurations would have $\frac{\left\langle H_{J_{p d}}\right\rangle}{J_{p d}} \in\left\{0, \pm \frac{1}{2}, \pm 1\right\}$, and can be ignored. This can be achieved using a 3SP-pair projector

$$
P_{3 S P}=\prod_{\Delta \lambda \in\left\{0, \pm \frac{1}{2}, \pm 1\right\}} \frac{\widehat{H}_{J_{p d}} / J_{p d}-\Delta \lambda}{-2-\Delta \lambda}
$$

wherein terms in the product vanish for the eigenvalues of the excluded levels, while scaling the relevant $\left\langle H_{J_{p d}}\right\rangle=-2 J_{p d}$ levels to unity. $P_{3 S P}$ thus projects out 

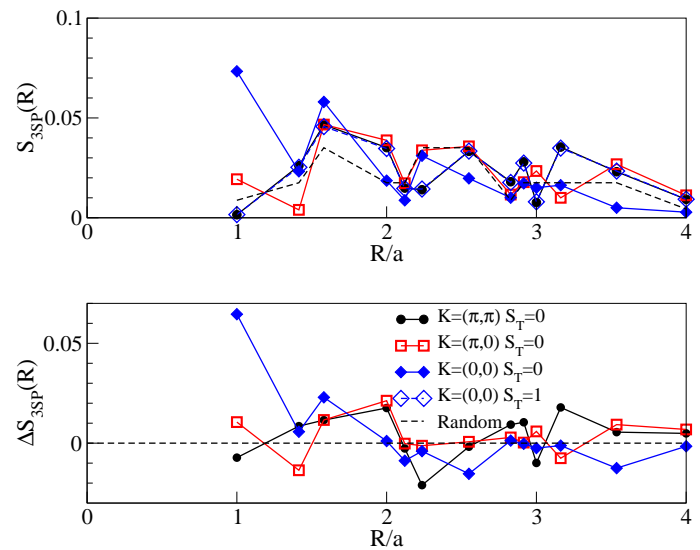

FIG. 6. Top: Probability of singlet 3SP pair, $\left\langle S_{3 S P}(R)\right\rangle$ for the lowest states in the $C_{S}=\frac{1}{2}$ subspace. Bottom: The difference from a randomly distributed paramagnetic configuration.

the subspace of having two 3SP's with any spin-spin correlation. All low energy states have $\gtrsim 0.78$ probability in the $P_{3 S P}$-projected subspace, roughly the square of single-hole solution's $\sim 0.9$ overlap with a $3 \mathrm{SP}, \underline{57}$ It is thus clear that the extra holes form three-spin polarons.

Section IV.A shows that the low-energy two-hole states are total spin $S=0$ singlets. It is thus interesting to check if the wavefunctions can be described simply by a $S=0$ AFM background plus a 3SP singlet pair, $\frac{1}{\sqrt{2}}(|\Uparrow\rangle|\Downarrow\rangle-|\Uparrow\rangle|\Downarrow\rangle)$. In Appendix B, we derive a numerical operator

$$
S_{3 S P}(R)=\sum_{|\eta|=R} s_{3 S P}(\eta)
$$

which measures the probability of having such a singlet as a function of charge-charge separation $R$, within the subspace projected by $P_{3 S P}$. The value is normalized with the analogous triplet measure, defined in a similar fashion:

$$
\sum_{R}\left(S_{3 S P}(R)+T_{3 S P}(R)\right)=1 .
$$

The singlet correlation should be compared to $\sum_{R} P^{\prime}(R)=1$, the random distribution of two 3SPs spread over the 64 oxygen sites but with no shared copper spin. The probability of singlet correlation is $\frac{1}{4}$ in a paramagnetic state. The difference of interest is then

$$
\Delta S_{3 S P}(R)=S_{3 S P}(R)-\frac{P^{\prime}(R)}{4} .
$$

Figure 6 shows $S_{3 S P}(R)$ and $\Delta S_{3 S P}(R)$ for the highsymmetry points eigenstates. It is evident that all states have enhanced short-range singlet nature as compared to the random distribution - a feature absent from previous studies using small clusters or the t-t'-J model which does not distinguish oxygen and copper sites. This short-range nature is not an artifact of small cluster squeezing the two polarons together because the cluster does allow the two holes to separate further apart, as confirmed by the long-range nature of charge correlation in Fig. 5 .

$\Delta S_{3 S P}(R)$ tells only part of the story because it is counter intuitive to have the two polarons with $R$ dependent singlet-triplet ratio as indicated by Fig. 6. The total spin is $S_{T}=0$ so the $N-4$ copper spins not neighbored by the oxygen charges must carry a spin of 1 when the two polarons tend to form a triplet. The 6-spin description, which is sufficient to describe the location of charge, is not sufficient to capture the spin carried by the polarons. The polarons produce a spin disturbance with a $R$-dependent spatial extent beyond the two copper spins sandwiching the oxygen charge. Note that the non-local spin disturbance around a polaron is already

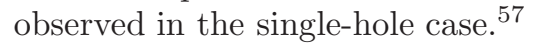

\section{DISCUSSION}

Section IV shows that in the GS, the two holes are not correlated spatially, since the probability $\Delta C(R)$ to find them at $R \leq 2 a$ is less than for randomly distributed holes. This suggests that if the binding energy indeed remains negative in the limit $N \rightarrow \infty$, the pair would have to be correlated in $\mathrm{k}$-space. One needs to be able to study larger clusters in order to fully settle this issue. However, local pairing correlations are seen for the two holes in the $\mathrm{S}=0, \mathrm{~K}=0$ state, showing that the competition between kinetic energy and a local attractive potential. For an energy cost of a single polaron's bandwidth, $\sim 2 J_{d d}$, the state changes from the $K=(\pi, \pi)$ delocalized GS to a $K=0 d_{x^{2}-y^{2}}$ local pair. This strong momentum dependency suggests that, amidst the presence of a local $d_{x^{2}-y^{2}}$ attraction, a local-pairing scenario cannot completely explain the full problem, at least within this model in the $n=2 / 32$ low-doping scenario.

Although we have broken the technological limit, two holes in a $32-\mathrm{CuO}_{2}$-unit-cell finite lattice cannot rigorously model all aspects of an infinite system. For example, the possibility of a quantum critical point in the $4.3 \%$ low-doping regime $\frac{65}{5}$ cannot be tested. There are other questions: what is the implication of the $\sim 2 J_{d d}$ penalty when long-range AFM order due to $J_{d d}$ is destroyed? Is there a better measure of the binding energy than referencing from a single polaron along with a AFM background? What are the translational and point-group symmetries of the overall wavefunction of more than two holes? Finite-size scaling beyond $\mathrm{N}=32$ would certainly provide a more robust description of the physics but would require more advanced approaches and better technologies.

Next we point out that the weak local attraction, if it ever gains prominence as doping is increased, would not lead to the unrealistic real-space clustering of holes as doping concentration is raised. The only hint of clustering mechanism is the attraction for local $d_{x^{2}-y^{2}}$ correlated holes as in the $K, S_{T}=0$ state. The nature 
of this attraction can be grasped from the $R \leq a$ local peaks of singlet tendency in Fig. 6. By expanding the 6-spin wavefunctions $\frac{1}{\sqrt{2}}(|\Uparrow\rangle|\Downarrow\rangle \pm|\Downarrow\rangle|\Uparrow\rangle)$ and taking the $\bar{S} \cdot \bar{S}$ between a copper spin from the first 3SP and one from the second 3SP, the expectation value is $-\frac{5}{18} J_{d d}$ if they are singlet and $+\frac{1}{18} J_{d d}$ if they are triplet. Two 3SPs can take advantage of this energy lowering by forming a singlet, but only if they are separated by an empty oxygen site which mediates an ordinary $\bar{S} \cdot \bar{S}$ Heisenberg AFM bond. The two-hole numerical solution indeed shows a local maximum when $R / a \leq 2$ (Fig. 6). Now, by expanding the 12-spin wavefunctions obtained as the direct product of two 3SP singlet pairs: $\frac{1}{2}(|\Uparrow\rangle|\Downarrow\rangle-|\Downarrow\rangle|\Uparrow\rangle) \otimes(|\Uparrow\rangle|\Downarrow\rangle-|\Downarrow\rangle|\Uparrow\rangle)$, the expectation value of $\bar{S} \cdot \bar{S}$ between a copper spin from the first pair and one from the second pair is exactly zero. Therefore, more than two holes cannot lower their energy by clustering.

Lastly, we point out features of the two-hole solution that is expected to prevail as the cluster size $N$ is increased. The doped charge would form a core of $3 S P$, which is surrounded by spin disturbances which determine the spin of the polaron, in agreement of the one-hole scenario 57 . The extent of spin disturbance varies with hole-hole separation. The R-dependent polaron-polaron correlations would not change drastically because the $N=32$ cluster can accommodate the two holes without artificially forcing them together as in the $N=16$ case. Due to this fact, the observed cross-over between locally and non-locally correlated states within $\mathrm{a} \sim 2 J_{d d}$ energy window is expected to be robust.

\section{CONCLUSIONS}

To summarize, we have extended our previous work ${ }^{57,58}$ to study two-hole states in $\mathrm{CuO}_{2}$ planes, in the context of the spin-polaron model of Ref. 57. Our numerical approach bypassed various technical limitations and extracted the explicit low-energy wavefunctions of two holes injected into a half-filled system with 32 copper and 64 oxygen sites.

The $N=32$ solution was found to be different from the $N=16$ solution. Similar to the $N=32$ two-hole tt'-J model, the GS was found at $K=(\pi, \pi)$, with $\Delta E \sim$ $\frac{1}{N} Q=(\pi, \pi)$ magnon excitation. The binding energy was found to be $(-0.091 \pm 0.272) J_{d d}$. In contrast to the $t-t^{\prime}-J$ model, we found the lowest state at $K=(0,0)$, without a $Q=(\pi, \pi)$ magnon, to have be a $d_{x^{2}-y^{2}}$ locally bound state.

Further analysis of the wavefunctions revealed that the charge carriers are 3SPs, $|\Uparrow\rangle$ and $|\Downarrow\rangle$, even in this multihole scenario, but the polarons' spin disturbances are extended in range, involving more than the two copper spins sandwiching the 3SP. From the correlation values, we established that the low-energy band results from the competition between kinetic energy and a local attractive potential which induces $d_{x^{2}-y^{2}}$ pair.
Lastly, we showed that real-space hole clustering is unlikely at higher concentration and also noted aspects that are expected to be robust for larger systems size. Study of higher-doping scenario could be interesting but would require more advanced technologies.

\section{ACKNOWLEDGMENTS}

We thank R. Thomale for discussions, P. W. Leung for sharing two-hole data for the t-t'-J model, I. Elfimov and Westgrid for tech support, and CFI, CIfAR, CRC and NSERC for funding.

\section{Appendix A: Implementation of the octapartite truncation for the AFM background}

First, we define singlet and triplet creation operators for the two oxygen holes, when located at sites $l \neq l^{\prime}$ :

$$
\begin{aligned}
s_{l, l^{\prime}}^{\dagger} & =\frac{1}{\sqrt{2}}\left(p_{l \uparrow}^{\dagger} p_{l^{\prime} \downarrow}^{\dagger}-p_{l \downarrow}^{\dagger} p_{l^{\prime} \uparrow}^{\dagger}\right) \\
t_{-1, l, l^{\prime}}^{\dagger} & =p_{l_{\downarrow}}^{\dagger} p_{l^{\prime} \downarrow}^{\dagger} \\
t_{0, l, l^{\prime}}^{\dagger} & =\frac{1}{\sqrt{2}}\left(p_{l \uparrow}^{\dagger} p_{l^{\prime} \downarrow}^{\dagger}+p_{l \downarrow}^{\dagger} p_{l^{\prime} \uparrow}^{\dagger}\right) \\
t_{1, l, l^{\prime}}^{\dagger} & =p_{l \uparrow}^{\dagger} p_{l^{\prime} \uparrow}^{\dagger} .
\end{aligned}
$$

Recall that $S$ is the total spin of the $N \mathrm{Cu}$ spins (the AFM background). According to quantum mechanical angular momentum addition, upon introducing the two doping holes, the $N+2$ spins can add up to a total spin $S_{T}$ by mixing a two-hole singlet with a background of total spin $S=S_{T}$, or by mixing a two-hole triplet with a background of total spin $S=S_{T}, S_{T} \pm 1$. Taking $S_{T}^{z}=0$, any spin background can be specified orthonormally with respect to a position $l$ :

$$
\square_{l, l^{\prime}}^{\dagger}|\alpha\rangle_{l} \equiv\left\{\begin{array}{c}
s_{l, l^{\prime}}^{\dagger}\left|\alpha, S^{z}=0\right\rangle_{l} \\
\sum_{z=-1}^{1} c\left(z, S, S_{T}\right) t_{z, l, l^{\prime}}^{\dagger}\left|\alpha, S^{z}=-z\right\rangle_{l}
\end{array}\right.
$$

Here, $\alpha$ denotes a particular group of $2 S+1$ spin configurations related by the total spin raising and lowering operators $S^{ \pm}=\sum_{l} S_{l}^{ \pm}$, summed over all $\mathrm{Cu}$ sites. The total spin of this $\alpha$ group can be $S=S_{T}$ for two-hole singlet and $S=S_{T}, S_{T} \pm 1$ for two-hole triplets. Due to the choice of the overall projection $S_{T}^{z}=0$, the two-hole singlet would mix only with backgrounds with $S^{z}=0$. The two-hole triplets would mix with three different projections $\left|\alpha, S^{z}=-1\right\rangle_{l},\left|\alpha, S^{z}=0\right\rangle_{l}$, and $\left|\alpha, S^{z}=1\right\rangle_{l}$ from the group $\alpha$. The weight $c\left(z, S, S_{T}\right)$ is the ClebschGordon coefficients for mixing these three states with the three two-hole triplets to achieve a state with total spin $S_{T}$ and $S_{T}^{z}=0$.

Exploitation of the translational symmetry is performed by the use of a Fourier series of the form

$$
\sim \sum e^{i K l} \square_{l+\epsilon, l^{\prime}+\epsilon^{\prime}}^{\dagger}|\alpha\rangle_{l}
$$


however, care must be taken to ensure orthonormality. Due to the commutation relation of the triplet and singlet (Eq. A1), the Fourier series is not straightforward for $\epsilon=\epsilon^{\prime}=\epsilon_{x / y}$ when both oxygen holes occupy $\mathrm{x}$ - or $\mathrm{y}$ rung oxygen orbitals (see Fig. 1a). The specification of these two-hole configurations requires the two orthogonal periodic lattice vector $L_{0}$ and $L_{1}$ of length $\sqrt{N}$ for the 2D, N-unit-cell lattice. Defining

$$
\delta l=\left(l_{0}^{\prime}-l_{0}, l_{1}^{\prime}-l_{1}\right)
$$

most hole-hole configurations can be classified in the re- gion

$$
\begin{aligned}
& 0 \leq \delta l_{0}<\frac{L_{0}}{2}, 0<\delta l_{1}<\frac{L_{1}}{2} \\
& 0<\delta l_{0}<\frac{L_{0}}{2}, \delta l_{1}=\frac{L_{1}}{2} \\
& 0<\delta l_{0}<\frac{L_{0}}{2},-\frac{L_{1}}{2}<\delta l_{1} \leq 0 \\
& \delta l_{0}=\frac{L_{0}}{2},-\frac{L_{1}}{2}<\delta l_{1}<0 .
\end{aligned}
$$

These states can be expressed using $N$-term Fourier series

$$
\left|\square_{x x / y y}, \delta l, \alpha, K\right\rangle=\frac{1}{\sqrt{N}} \sum e^{i K l} \square_{l+\epsilon_{x / y}, l+\delta l+\epsilon_{x / y}}^{\dagger}|\alpha\rangle_{l}
$$

Because the two oxygen holes are indistinguishable fermions (Eq. A1), there are three remaining $\delta l$ values which require special attention due to the periodic boundary condition.

$$
\begin{gathered}
\delta l_{0}=\frac{L_{0}}{2}, \delta l_{1}=0 \\
\delta l_{0}=0, \delta l_{1}=\frac{L_{1}}{2} \\
\delta l_{0}=\frac{L_{0}}{2}, \delta l_{1}=\frac{L_{1}}{2}
\end{gathered}
$$

The number of terms in the Fourier series depends on the spin background translated by $\delta l: T_{\delta l}|\alpha\rangle_{l}$. If such a translation yields an orthogonal state, ${ }_{l}\left\langle\alpha\left|T_{\delta l}\right| \alpha\right\rangle_{l}=0$, the Fourier series still has $N$ terms. For the example of $\delta l=\left(\frac{L_{0}}{2}, 0\right)$,

$$
\begin{aligned}
\left|\square_{x x / y y}, \delta l, \alpha, K\right\rangle & =\frac{1}{\sqrt{N}} \sum e^{i K l} \square_{l+\epsilon_{x / y}, l+\delta l+\epsilon_{x / y}}^{\dagger}|\alpha\rangle_{l} \\
& =\frac{1}{\sqrt{N}} \sum_{l_{1}=0}^{L_{1}-1} e^{i K_{1} l_{1}} \sum_{l_{0}=0}^{\frac{L_{0}}{2}-1} e^{i K_{0} l_{0}} \square_{l+\epsilon_{x / y}, l+\delta l+\epsilon_{x / y}}^{\dagger}\left(1+s_{\square} e^{i K_{0} \frac{L_{0}}{2}} T_{\frac{L_{0}}{2}}\right)|\alpha\rangle_{l},
\end{aligned}
$$

where $s_{\square}$ is the sign change due to hole-swapping in the singlet or triplet $\left(\square_{a, b}^{\dagger}=s_{\square} \square_{b, a}^{\dagger}\right)$. For the case of $\left\langle\alpha\left|T_{\delta l}\right| \alpha\right\rangle_{l}= \pm 1$, the above expansion makes clear that there can be only $\frac{N}{2}$ terms in the Fourier series due to the term $\left(1+s_{\square} e^{i K_{0} \frac{L_{0}}{2}} T_{\frac{L_{0}}{2}}\right)$. For this case the series has the form

$$
\left|\square_{x x / y y}, \delta l, \alpha, K\right\rangle=\sqrt{\frac{2}{N}} \sum_{l_{1}=0}^{L_{1}-1} e^{i K_{1} l_{1}} \sum_{l_{0}=0}^{\frac{L_{0}}{2}-1} e^{i K_{0} l_{0}} \square_{l+\epsilon_{x / y}, l+\delta l+\epsilon_{x / y}}^{\dagger}|\alpha\rangle_{l} .
$$

The formulation for the case where one hole occupies $p_{l+\epsilon_{x}}$ and the other $p_{l+\epsilon_{y}}$ is straightforward. All values of $\delta l$ are unique and the Fourier series has the form

$$
\left|\square_{x y}, \delta l, \alpha, K\right\rangle=\frac{1}{\sqrt{N}} \sum e^{i K l} \square_{l+\epsilon_{x}, l+\delta l+\epsilon_{y}}^{\dagger}|\alpha\rangle_{l} .
$$


Therefore, a full orthonormal Hilbert space can be specified by the states $\left|\square_{x y}, \delta l, \alpha, K\right\rangle$ and $\left|\square_{x x / y y}, \delta l, \alpha, K\right\rangle$. Translational symmetry is specified by the quantum number K. Total spin and its projection are specified by the singlet/triplet nature of the two oxygen holes $(\square)$ (singlet-triplet) in conjunction to the spin background $\alpha$.

The above formulation allows the enumeration of different oxygen-oxygen configurations for the two doping holes, for a given $|\alpha\rangle$ background. An enumeration of the different $|\alpha\rangle$ states is now the missing step for computation. This is not a trivial exercise because the computation for large $N$ requires that a large number of possible $|\alpha\rangle$ states be discarded according to the truncation criterion of Eq. 15 Note that this criterion is based on the total spin of each sublattice, and the basis $\alpha$ is built according to quantum mechanical spin addition.

An arbitrary enumeration of $|\alpha\rangle$ basis would not yield an efficient computation because of several challenges. First, the truncation of less important states should be controllable systematically and flexible enough to adapt to the many unknowns of doped systems. Second, within the truncated basis transformed away from the natural $z$-projected description, there should be a fast way of indexing initial and final states upon Hamiltonian operations. Third, one should have apriori knowledge about the identically-zero matrix elements in order to avoid them for sparse matrices' performance scaling.

The above formulation specifies a basis state in terms of a Fourier sum over a particular reference site $l$. The spin background $|\alpha\rangle_{l}$ and one oxygen hole $p_{l+\epsilon, \sigma}^{\dagger}$ are referenced at $l$ while the other oxygen hole is located at $\delta l$ away from the reference oxygen hole. The hopping terms $T_{p p}, T_{\text {swap }}$ and $J_{p p}$ operate on both oxygen holes. Hopping of the non-reference oxygen hole would not change the reference point $l$; however, hopping of the oxygen hole at $l+\epsilon$ would yield a state with no oxygen at $l+\epsilon$. This simply means that the final state can be specified at a new referencing position $l^{\prime}$ with a phase shift of $e^{i K\left(l-l^{\prime}\right)}$, and that the new spin background is obtained by a translation $\left|\alpha^{\prime}\right\rangle_{l^{\prime}}=\widehat{\operatorname{Tr}}\left(l-l^{\prime}\right)|\alpha\rangle_{l}$. It is thus advantageous to build the $|\alpha\rangle_{l}$ spin basis such that the construction is the same upon translation.

The octapartite approach introduced in Ref. 58 satisfies all of the above requirements. First, the copper spins are divided into eight groups as shown in Fig. 7 For each group of $\frac{N}{8}$ spins, we start with the $z$-projected representation of $2^{\frac{N}{8}}$ states and transform into a ClebschGordan basis $\left|s_{\frac{N}{8}}, s_{\frac{N}{8}}^{z}\right\rangle$. Each $z$-projected basis state is represented using bits of an unsigned integer and each Clebsch-Gordan series is stored as a sparse vector. With a particular enumeration of $\left|s_{\frac{N}{8}}, s_{\frac{N}{8}}^{z}\right\rangle$ states, we mix two identical enumerations to build $\left|s_{\frac{N}{4}}, s_{\frac{N}{4}}^{z}\right\rangle$ according to Clebsch-Gordan addition. Then with two identical enumerations of $\left|s_{\frac{N}{4}}, s_{\frac{N}{4}}^{z}\right\rangle$, we build a single enumeration of $\left|s_{\frac{N}{N}}, s_{\frac{N}{N}}^{z}\right\rangle$. Finally, we can similarly enumerate the over-

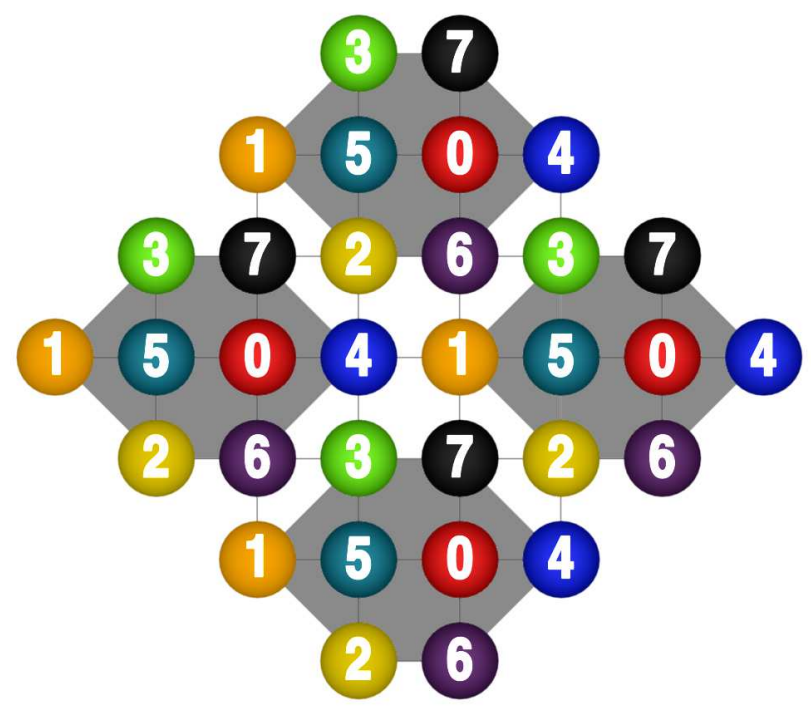

FIG. 7. $N=32$ cluster divided into eight groups labbeled $0=$ 7. The spins within each group are connected by multiples of $(2 a, \pm 2 a)$. Spins from a particular group are always in the same environment, eg 1 always 4,5,6 and 7 as neighbors.

all $|\alpha\rangle$ background as $\left|s_{N}, s_{N}^{z}\right\rangle$ from the enumeration of $\left|s_{\frac{N}{2}}, s_{\frac{N}{2}}^{z}\right\rangle$. This is a recursive enumeration procedure. At each stage, the states are indexed by a non-negative integer, state_index. The state represented by a particular index value can be derived from the "parent" basis with half the number of spins using, for example, the following loop structure:

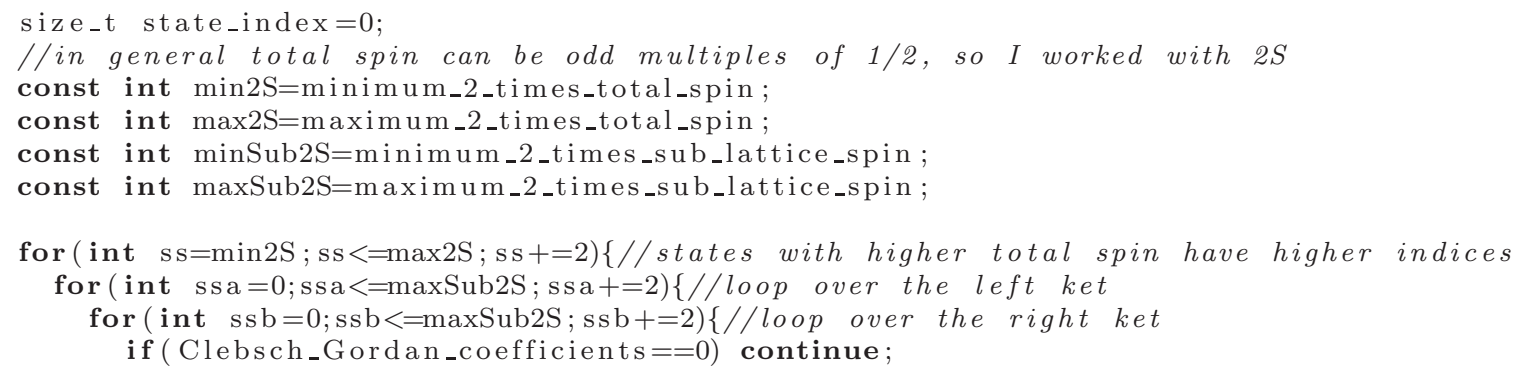




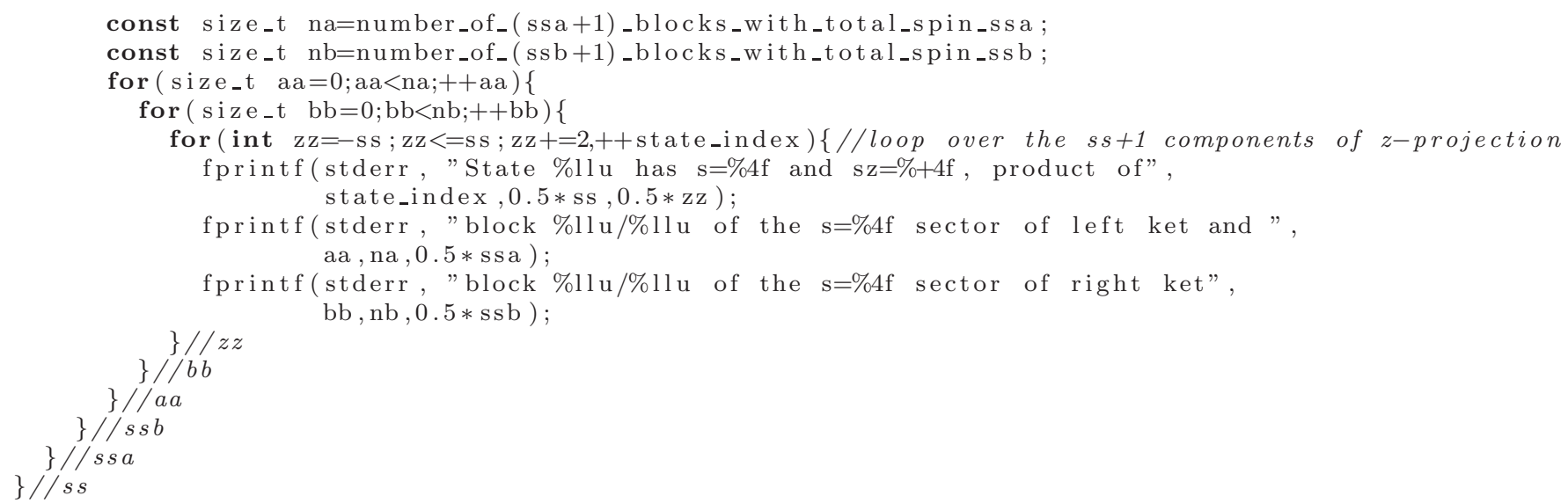

The benefit of this enumeration is an instantaneous "reverse lookup". When performing Hamiltonian operations, one is really interested in the non-zero overlap between the outgoing states and those in the orthonormal basis. The naive way is to compute the dot product against all basis states, but this adds a $O(n)$ layer on top everything else and is detrimental in the case of large systems. Under the above looping scheme, an increasing state_index is associated with increasing values of ssa, aa, ssb, and bb. Because the index shift for states of these combinations is known a priorily, any arbitrary ket $\left|\sigma_{a}, \sigma_{a}^{z}\right\rangle\left|\sigma_{b}, \sigma_{b}^{z}\right\rangle$, is trivially associated with these four indices so states with non-zero overlap are known immediately, with an $O(1)$ reverse lookup operation. Nonzero matrix elements can thus be computed efficiently by changing ssa, aa, ssb, and bb before the reverse lookup. The neighboring pattern in Fig. 7 eases the determination of spin backgrounds upon translation. The determination of matrix elements is trivially parallelizable. It is apparent that truncation according to the criterion of Eq. 15 can be performed at last stage of mixing by simply tuning the value of minSub2S.

\section{Appendix B: Singlet correlator between two three-spin polarons}

If we consider only the 6 spins involved in the two 3SPs, the projected wavefunction is a superposition of the four possible 3SP pairs:

$$
\begin{aligned}
\left|\phi_{6}\right\rangle= & a \frac{|\Uparrow\rangle|\Downarrow\rangle-|\Uparrow\rangle|\Downarrow\rangle}{\sqrt{2}}+b|\Uparrow\rangle|\Uparrow\rangle \\
& +c \frac{|\Uparrow\rangle|\Downarrow\rangle+|\Uparrow\rangle|\Downarrow\rangle}{\sqrt{2}}+d|\Downarrow\rangle|\Downarrow\rangle .
\end{aligned}
$$

Defining the oxygen-oxygen singlet operator for the two oxygen spins, $p_{1, \sigma}^{\dagger}$ and $p_{2, \sigma}^{\dagger}$

$$
s_{1,2}^{\dagger}=\frac{1}{\sqrt{2}}\left(p_{1, \uparrow}^{\dagger} p_{2, \downarrow}^{\dagger}-p_{1, \downarrow}^{\dagger} p_{2, \uparrow}^{\dagger}\right),
$$

the probability of finding a oxygen-oxygen singlet in $\left|\phi_{6}\right\rangle$ is

$$
\left\langle s_{1,2}^{\dagger} s_{1,2}\right\rangle=\frac{3}{9} a^{2}+\frac{2}{9}\left(b^{2}+c^{2}+d^{2}\right) .
$$

Solving for $a^{2}$ and generalizing the expression to different hole-hole separations $\eta$, the singlet nature of the 3SP pair when at distance $\eta$ apart is gauged by

$$
s_{3 S P}(\eta)=\frac{\left\langle P_{3 S P}(9 \hat{s}(\eta)-2 \hat{c}(\eta)) P_{3 S P}\right\rangle}{\left\langle P_{3 S P} \sum_{\eta^{\prime}} \hat{c}\left(\eta^{\prime}\right) P_{3 S P}\right\rangle}
$$

where

$$
\hat{s}(\eta)=\sum_{l, \epsilon} s_{l+\epsilon, l+\epsilon+\eta}^{\dagger} s_{l+\epsilon, l+\epsilon+\eta}
$$

measures the probability of the two $\mathrm{O}$ holes to be in a singlet at distance $\eta$ apart, irrespective of what the $\mathrm{Cu}$ spins are doing. Thus, $s_{3 S P}(\eta)$ is the probability, within the projected subspace, of the two oxygen holes and the AFM background to cooperate to form a 3SP singlet pair separated by a distance $\eta$. The value ranges from zero for no singlet nature to unity for pure singlet at this holehole separation; however, these two extreme values are not possible due to, for example, the spatial spreading required to lower the kinetic energy of $T_{p p}$ and $T_{\text {swap }}$. The measure can be summed as a function of hole-hole separation

$$
S_{3 S P}(R)=\sum_{|\eta|=R} s_{3 S P}(\eta)
$$

which is normalized with the analogous triplet measure, defined in a similar fashion:

$$
\sum_{R}\left(S_{3 S P}(R)+T_{3 S P}(R)\right)=1 .
$$

The singlet correlation should be compared to $\sum_{R} P^{\prime}(R)=1$, the random distribution of two 3SPs 
spread over the 64 oxygen sites but with no shared copper spin. The probability of singlet correlation is $\frac{1}{4}$ in a paramagnetic state. The difference of interest is then

$$
\Delta S_{3 S P}(R)=S_{3 S P}(R)-\frac{P^{\prime}(R)}{4} .
$$

1 E. Manousakis, Rev. Mod. Phys. 63, 1 (1991).

2 J. G. Bednorz and K. A. Mller, Zeitschrift fr Physik B Condensed Matter 64(2) 189 (1986)

3 J. Zaanen, S. Chakravarty, T. Senthil, P. W. Anderson, P. A. Lee, J Schmalian, M. Imada, D. Pines, M. Randeria, C. Varma, M. Vojta, and M. Rice, Nature Physics 2138 (2006)

4 D. A. Bonn, Nature Phys. 2, 159 (2006)

5 S. Hufner, M. A. Hossain, A. Damascelli, and G. A. Sawatzky, Rep. Prog. Phys. 71, 062501 (2008)

${ }^{6}$ D. M. Newns and C. C. Tsui, Nature Phys. 3, 184 (2007)

7 G. Sangiovanni, O. Gunnarsson, E. Koch, C. Castellani, and M. Capone, Phys. Rev. Lett. 97, 046404 (2006)

8 C. M. Varma, Phys. Rev. B 5514554 (1997)

${ }^{9}$ Cdric Weber, Andreas Luchli, Frdric Mila, and Thierry Giamarchi, Phys. Rev. Lett. 102, 017005 (2009).

10 C. Varma, Nature (London) 468, 184 (2010).

11 V. Cataudella, G. De Filippis, A. S. Mishchenko, and N. Nagaosa, Phys. Rev. Lett 99, 226402 (2007).

12 M. Ogata and H. Fukuyama, Rep. Prog. Phys. 71, 036501 (2008)

13 P. A. Lee, Rep. Prog. Phys. 71, 012501 (2008).

14 P .A. Lee, N. Nagaosa, and X. G. Wen, Rev. Mod. Phys. 78, 17 (2006)

15 F.C. Zhang and T.M. Rice, Phys. Rev. B 37, 3759 (1988).

16 P. W. Leung, B. O. Wells, and R.J. Gooding, Phys. Rev. B 56, 6320 (1997).

17 A. F. Barabanov, A. V. Mikheenkov and A. M. Belemuk, JETP Lett. 75, 107 (2002).

${ }^{18}$ E Gull A J Millis, A I Lichtenstein, A N Rubtsov, M. Troyer, and P Werner, Rev. Mod. Phys. 83349 (2011)

19 P. W. Leung, Phys. Rev. B 65205101 (2002); also in private communication.

20 J. Zaanen and A. M. Oles, Phys. Rev. B 37, 9423 (1988)

21 D.M. Frenkel, R.J. Gooding, B.I. Shraiman, and E.D. Siggia, Phys. Rev. B 41, 350 (1990)

22 J.L. Shen and C.S. Ting, Phys. Rev. B 41, 1969 (1990)

23 H.Q. Ding, G.H. Lang, and W.A. Goddard, Phys. Rev. B 46, 14317 (1992)

24 Y. Petrov and T. Egami, Phys. Rev. B 58, 9485 (1998).

25 A. Macridin, M. Jarrell, Th. Maier, and G.A. Sawatzky, Phys. Rev. B 71, 134527 (2005)

26 J.F. Annett and R. M. Martin, Phys. Rev. B 42, 3929 (1990)

27 R. Eder and K. W. Becker, Z. Phys. B. 79, 333 (1990)

28 V. J. Emery, Phys. Rev. Lett. 58, 2794 (1987)

29 H. Eskes and G. A. Sawatzky, Phys. Rev. Lett. 61, 1415 (1988).

30 M. Greiter and R. Thomale, Phys. Rev. Lett. 99, 027005 (2007)

31 R. Thomale and M. Greiter, Phys. Rev. B 77094511 (2008)

${ }^{32}$ L. Klein and A. Aharony, Phys. Rev. B 45, 9915 (1992).

33 D. F. Digor, P. Entel, V. A. Moskalenko and N. M. Plakida, Theor. Math. Phys. 149, 1382 (2006)

${ }^{34}$ L. Hozoi, S. Nishimoto, G. Kalosakas, D. B. Bodea, and S.
Burdin, Phys. Rev. B 75, 024517 (2007)

35 C.H. Patterson, Phys. Rev. B 77, 094523 (2008)

${ }^{36}$ L. Hozoi, M.S. Laad, and P. Fulde, Phys. Rev. B 78, 165107 (2008).

37 C Weber, K Haule, G Kotliar Nature Physics 6, 574-578 (2010)

38 C Weber, K Haule, G Kotliar, Phys. Rev. B 82, 125107 (2010)

39 T. Yanagisawa, S. Koike and K. Yamaji, Phys. Rev. B 64 184509 (2001)

40 J. Bonca, S. Maekawa, and T. Tohyama, Phys. Rev. B 76, 035121 (2007)

41 F. Tan and Q.H. Wang, Phys. Rev. Lett. 100, 117004 (2008).

42 M. Merz, N. Ncker, P. Schweiss, S. Schuppler, C. T. Chen, V. Chakarian, J. Freeland, Y. U. Idzerda, M. Klser, G. Mller-Vogt, and Th. Wolf, Phys. Rev. Lett. 805192 (1998)

43 R. Schuster, S. Pyon, M. Knupfer, J. Fink, M. Azuma, M. Takano, H. Takagi, and B. Bchner, Phys. Rev. B 79, 214517 (2009).

44 M. Vojta, Adv. Phys. 58, 699 (2009).

45 P. Abbamonte, A. Rusydi, S. Smadici, G. D. Gu, G. A. Sawatzky, and D. L. Feng, Nature Phys. 1, 155 (2005).

${ }^{46}$ M. J. Lawler, K. Fujita, Jhinhwan Lee, A.R. Schmidt, Y. Kohsaka, Chung Koo Kim, H. Eisaki, S. Uchida, J.C. Davis, J.P. Sethna, and Eun-Ah Kim, Nature (London) 466, 347 (2010).

47 G. Yu, Y. Li, E. M. Motoyama, X. Zhao, N. Bariic', Y. Cho, P. Bourges, K. Hradil, R. A. Mole, and M. Greven, Phys. Rev. B 81, 064518 (2010).

48 Yuan Li, V. Baldent, G. Yu, N. Bariic', K. Hradil, R. A. Mole, Y. Sidis, P. Steffens, X. Zhao, P. Bourges, M. Greven, Nature (London) 468, 283 (2010).

49 B. Fauque et al., Phys. Rev. Lett. 96, 197001 (2006).

50 D C Peets, D G Hawthron, K M Shen, Y J Kim, D S Ellis, H Zhang, S Komiya, Y Ando, G A Sawatzky, R Liang, D A Bonn, and W N Hardy, Phys. Rev. Lett. 103087402 (2009)

51 J. Zaanen, G.A. Sawatzky and J.W. Allen, Phys. Rev. Lett. 55, 418 (1985).

52 P. W. Anderson, Phys. Rev. 115, 2 (1959).

53 J.H. Jefferson, H. Eskes, and L.F. Feiner, Phys. Rev. B. 45, 7959 (1992)

54 O. P. Sushkov, G. A. Sawatzky, R. Eder, and H. Eskes, Phys. Rev. B. 56, 11769 (1997).

${ }^{55}$ K. M. Shen, F. Ronning, D. H. Lu, W. S. Lee, N. J. C. Ingle, W. Meevasana, F. Baumberger, A. Damascelli, N. P. Armitage, L. L. Miller, Y. Kohsaka, M. Azuma, M. Takano, H. Takagi, and Z.-X. Shen, Phys. Rev. Lett. 93, 267002 (2004)

${ }^{56}$ K. M. Shen, F. Ronning, W. Meevasana, D. H. Lu, N. J. C. Ingle, F. Baumberger, W. S. Lee, L. L. Miller, Y. Kohsaka, M. Azuma, M. Takano, H. Takagi, and Z.-X. Shen, Phys Rev B 75, 075115 (2007).

57 B. Lau, M. Berciu and G. A. Sawatzky, Phys. Rev. Lett. 
106036401 (2011).

58 B. Lau, M. Berciu and G. A. Sawatzky, Phys Rev B 81, 172401 (2010).

59 V. J. Emery and G. Reiter, Phys. Rev. B 38, 4547 (1988).

60 B. I. Kochelaev, J. Sichelschmidt, B. Elschner, W. Lemor, and A. Loidl, Phys. Rev. Lett. 794274 (1997)
61 D. D. Betts, H.-Q.Lin and J. S. Flynn, Can. J. Phys. 77, 353 (1999).

62 M. Berciu and S. John, Phys. Rev. B 6116454 (2000).

63 P. Fulde and R. A. Ferrell, Phys. Rev. 135 A550 (1964).

64 A. I. Larkin and Yu. N. Ovchinnikov, Sov. Phys. JETP 20 $762(1965)$

65 S-P Kou and Z-Y Weng, Phys. Rev. Lett. 90157003 (2003) 